\title{
Épidémiologie des norovirus et de la gastroentérite virale en Ontario, Canada, 2009 à 2014
}

\author{
Stephanie L Hughes ${ }^{1 *}$, Amy L Greer ${ }^{1}$, Alex J Elliot ${ }^{2}$, Scott A McEwen'1 Ian Young ${ }^{3}$, \\ Andrew Papadopoulos ${ }^{1}$
}

\section{Résumé}

Contexte : Le norovirus est la cause la plus courante de gastroentérite aiguë au Canada. La maladie cause une grande morbidité et des coûts sociétaux élevés. L'objectif de cet article est de décrire l'épidémiologie des norovirus dans la province de l'Ontario, au Canada, de 2009 à 2014.

Méthodes : Pour évaluer l'activité des norovirus et de la gastroentérite virale en Ontario, trois ensembles de données ont été acquis auprès du gouvernement provincial, soit deux ensembles de données de surveillance traditionnelles (éclosions et laboratoires) et des données de surveillance syndromique (télésanté), tous couvrant la période de 2009 à 2014. Toutes les éclosions, les demandes de laboratoire et les appels de télésanté ont d'abord été évalués pour le nombre total de gastroentérites virales. On a calculé les totaux de norovirus et de maladies qui semblent provenir des norovirus sous forme de proportion de gastroentérite virale afin d'estimer les niveaux d'activité propres aux agents. Les types d'établissements touchés, les sexes et les groupes d'âge ont également été analysés.

Résultats : Entre 2009 et 2014, 41,5\% des éclosions de gastroentérite virale, 63,4 \% des soumissions aux laboratoire de gastroentérite virale et $36,6 \%$ de tous les appels à la télésanté liés à la gastroentérite aiguë (non limités aux causes virales) ont été attribués aux norovirus et aux maladies qui semblent provenir des norovirus en Ontario. Le type d'établissement le plus souvent touché était celui des foyers de soins de longue durée et les groupes d'âge les plus touchés étaient les plus jeunes (moins de cinq ans) et les plus âgés (plus de 65 ans). Les femmes étaient légèrement plus touchées que les hommes.

Conclusion : Les norovirus et les maladies qui semblent provenir des norovirus ont été la principale cause de gastroentérite virale en Ontario entre 2009 et 2014. Ils représentaient le pourcentage le plus élevé de gastroentérite virale par rapport à tous les autres virus associés aux gastroentérites virales. Des travaux supplémentaires sont nécessaires pour déterminer tous les coûts des composantes et les mesures de santé publique nécessaires pour réduire le fardeau de la maladie.
Cette oeuvre est mise à la disposition selon les termes de la licence internationale Creative Commons Attribution 4.0

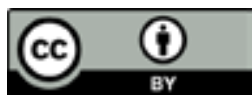

Affiliations

1 Département de médecine des populations, Université de Guelph, Guelph, ON

2 Équipe de surveillance syndromique en temps réel, Service de terrain, National Infection Service, Public Health England, Birmingham, Royaume-Uni

${ }^{3}$ École de santé professionnelle et publique, Université Ryerson, Toronto, ON

*Correspondance :

shughes@alumni.uoguelph.ca

Citation proposée : Hughes SL, Greer AL, Elliot AJ, McEwen SA, Young I, Papadopoulos, A. Épidémiologie des norovirus et de la gastroentérite virale en Ontario, Canada, 2009 à 2014. Relevé des maladies transmissibles au Canada 2021;47(10):442-9. https://doi.org/10.14745/ccdr.v47i10a01f

Mots-clés : norovirus, gastroentérite virale, gastroentérite aiguë, surveillance, Ontario, Canada

\section{Introduction}

Le norovirus est la cause la plus courante de gastroentérite infectieuse en Ontario, au Canada (1-3). Il comprend environ $50 \%$ de la gastroentérite aiguë (toutes les étiologies) (4). Son taux de morbidité élevé est dû à sa faible dose infectieuse (environ 18 à 1000 particules virales), à ses diverses voies de transmission, à son excrétion virale prolongée, à son immunité de courte durée et à sa persistance dans l'environnement $(5,6)$. Sa transmission efficace lui permet de prospérer dans des régions où la population est concentrée, comme les navires de croisière et les maisons de retraite $(1,7)$. Le fardeau de la maladie est élevé, avec environ 3,4 millions de cas et des dépenses hospitalières de 21 millions de dollars canadiens par année au Canada $(2,8)$. On estime également qu'aux États-Unis, une personne connaîtra en moyenne cinq épisodes de norovirus au cours de sa vie (9).

La maladie se caractérise par l'apparition soudaine de nausées, de vomissements, de diarrhée, de crampes abdominales et de malaises et se transmet par la voie fécale-orale et particules aérosolisés des vomissements $(1,5)$. La période d'incubation est 
courte (environ 10 à 48 h) et les symptômes sont généralement disparus en un à trois jours; toutefois, cette période est souvent plus longue chez les personnes à risque élevé, comme les très jeunes et les personnes âgées $(1,5)$. La maladie est habituellement traitée en clinique externe (10), bien qu'il puisse y avoir des séquelles et des effets secondaires graves, comme le syndrome du côlon irritable, une entérocolite nécrosante ou la mort $(1,11)$.

Les enfants sont particulièrement vulnérables à la maladie, nécessitant des soins médicaux plus fréquemment que tout autre groupe d'âge $(10,12)$. Cela souligne le besoin de surveillance pour informer la santé publique, planifier des mesures d'intervention appropriées et mettre au point des vaccins (13). L'absence de mécanismes officiels de déclaration des cas de norovirus (et de la gastroentérite aiguë en général) entraîne des lacunes dans les connaissances. Environ $15 \%$ des personnes atteintes de gastroentérite aiguë consultent un médecin et, de ce nombre, on demande des échantillons diagnostiques sur seulement $13 \%$ des cas (14).

Dans cette étude, l'épidémiologie des norovirus dans la province de l'Ontario, au Canada, est décrite à l'aide de données confirmées sur les éclosions, de données de tests en laboratoire et des appels de télémédecine avec des cas de patients qui vomissent.

\section{Méthodes}

Cette étude a été menée à partir de données de la province canadienne de l'Ontario, qui comptait une population d'environ 14,3 millions de résidents au moment de la présente étude (15).

\section{Ensembles de données}

Toutes les données acquises et utilisées dans cette étude ont été rendues anonymes (aucun identifiant personnel). Pour de plus amples renseignements, veuillez consulter l'appendice.

Éclosions : L'ensemble de données du Système intégré d'information sur la santé publique (SIISP) représente les éclosions confirmées de gastroentérite virale dans les établissements de l'Ontario signalées aux bureaux de santé publique locaux.

Rapports de laboratoire : L'ensemble de données des laboratoires de Santé publique Ontario représente tous les échantillons soumis à Santé publique Ontario dont on soupçonne la présence de gastroentérite virale à des fins d'analyse de confirmation; plus précisément, cet ensemble de données contient tous les échantillons envoyés à Santé publique Ontario dont on soupçonne la présence de norovirus ou de rotavirus.

Appels de télésanté : L'ensemble de données de Télésanté Ontario représente tous les appels faits au service de télésanté de la province dont le motif de consultation est lié à un problème gastro-intestinal. Ces appels liés à un problème gastro-intestinal représentent un ensemble de symptômes de type gastroentérite aiguë, englobant une portée plus large que les appels sur les gastroentérites virales, enregistrés par le personnel infirmier de Télésanté Ontario. Les appelants peuvent présenter ces symptômes gastro-intestinaux pour diverses raisons, dont les norovirus. Par conséquent, les appels de télésanté avec les motifs de consultations " vomissements » et « vomissements avec diarrhée » principalement sélectionnés ont été choisis comme les " motifs principaux liés aux vomissements " et utilisés comme indicateur de l'activité des cas de norovirus dans cette étude. Le motif de consultation principal concernant les vomissements a été choisi en raison de son respect des principaux symptômes de la maladie liée aux norovirus et des données probantes d'études antérieures démontrant son rôle en tant qu'indicateur de la maladie $(1,5,16)$.

\section{Analyses de données}

Les trois ensembles de données de cette étude ont été analysés pour les éclosions totales de gastroentérite virale et la proportion attribuée aux norovirus (ou, dans le cas des données de Télésanté Ontario, la maladie gastroentérite due à l'incapacité de confirmer la présence de norovirus). Ces pourcentages ont été utilisés pour évaluer les niveaux d'activité des norovirus en Ontario. Les données du recensement de l'Ontario, ainsi que le nombre total de types d'établissements (garderies, foyers de soins de longue durée, maisons de retraite, établissements correctionnels) ont servi de dénominateurs pour normaliser certaines analyses (17-20).

Des analyses descriptives ont été effectuées à l'aide de SAS v.9.4 (Cary, Caroline du Nord, États-Unis) et de Microsoft Office (Excel) 2010 (Redmond, Washington, États-Unis).

\section{Résultats}

Éclosions : Il y a eu 3100 éclosions de gastroentérite virale en Ontario au cours des années 2009 à 2014; 41,6 \% ont été causées par des norovirus, soit par la définition de cas ou la confirmation en laboratoire. Les $58,4 \%$ restants ont été attribués à des adénovirus, des astrovirus, des entérovirus/échovirus, des rotavirus, d'autres calicivirus et des gastroentérites non spécifiées ou autres.

Entre 2009 et 2014, 45,1 \% des éclosions de gastroentérite virale se sont produites dans des foyers de soins de longue durée, $30,9 \%$ dans des garderies, $22,6 \%$ dans des maisons de retraite, $0,3 \%$ dans des établissements correctionnels et $1,2 \%$ dans d'autres contextes. Cette répartition est demeurée relativement constante lorsque l'analyse a été limitée aux éclosions de norovirus, auquel cas les maisons de retraite ont remplacé les garderies comme deuxième type d'établissement le plus fréquemment touché. De ces éclosions de gastroentérite 
virale dans les foyers de soins de longue durée, plus de la moitié $(57,2 \%)$ ont été attribuées à des norovirus. La figure 1 présente une ventilation institutionnelle des éclosions de norovirus.

Figure 1 : Pourcentage d'établissements touchés par des éclosions de norovirus en Ontario, de 2009 à 2014

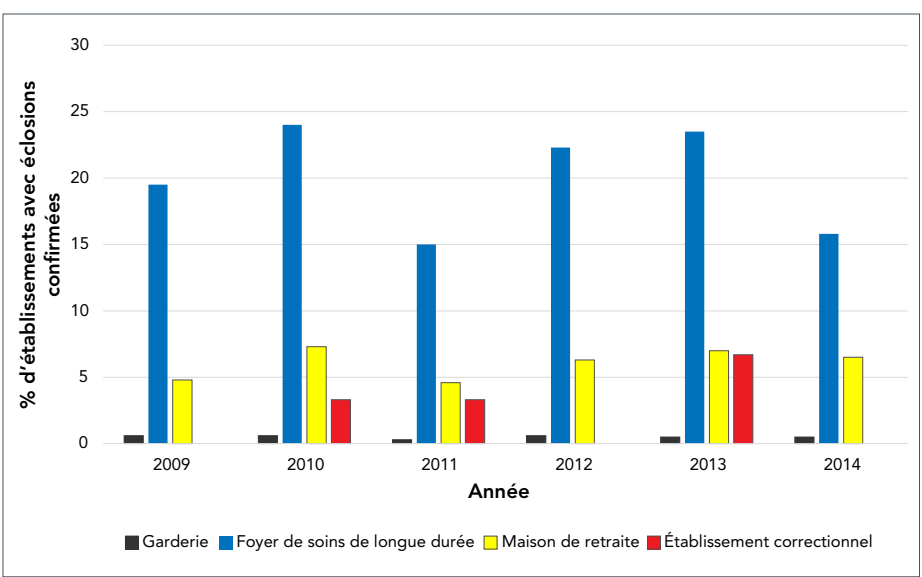

Le nombre d'éclosions de norovirus par année a été relativement stable au cours des six dernières années : 211 en 2009, 265 en 2010, 178 en 2011, 247 en 2012, 215 en 2013 et 175 en 2014.

Cela est comparable à la stabilité des éclosions de gastroentérite virale au cours de la même période.

La distribution saisonnière des éclosions de norovirus par mois et par année est illustrée à la figure 2. La durée moyenne des éclosions de gastroentérite virale était de 12,6 jours (variant de 1 à 78 jours) et de 14,1 jours (variant de 1 à 52 jours) pour les éclosions de norovirus.

Figure 2 : Saisonnalité des soumissions d'échantillons ${ }^{\mathrm{a}}$ de norovirus en laboratoire et des éclosions ${ }^{b}$ en Ontario selon le mois et l'année, de 2009 à 2014

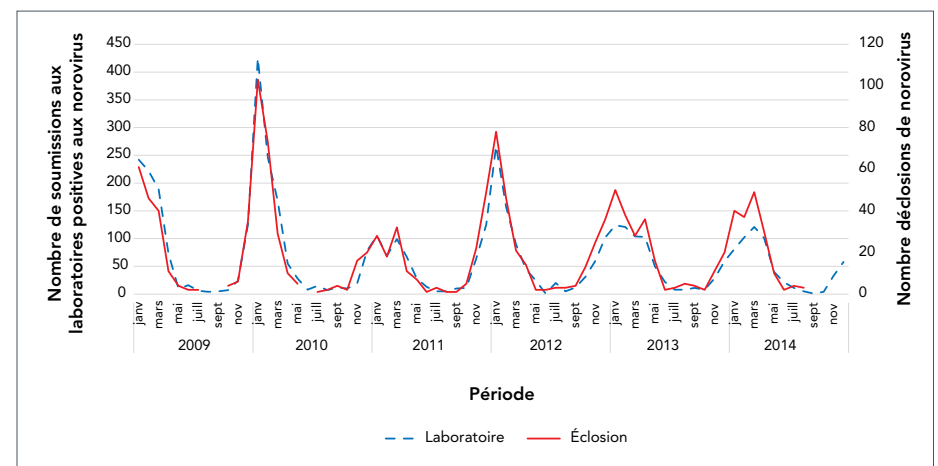

a Les soumissions au laboratoire ont été confirmées à l'aide des données des Laboratoires de Santé publique Ontario

b Les éclosions ont été confirmées à l'aide des données du Système intégré d'information sur la santé publique (SIISP)

Rapports de laboratoire : Entre 2009 et 2014 inclusivement, 29459 échantillons de rotavirus et de gastroentérite virale de type norovirus ont été soumis à SPO. La majorité ( $n=22$ 147; 75,2 \%) était négative. Parmi les échantillons positifs ( $n=7312$ ), 63,4 \% ont été attribués à des norovirus, les $36,6 \%$ restants étant composés de diverses autres étiologies de gastroentérite virale, y compris l'adénovirus (16,5\%), l'astrovirus $(1,1 \%)$, d'autres calicivirus $(0,3 \%)$, les picornavirus $(0,3 \%)$, le rotavirus $(81,0 \%)$ ou le sapovirus $(0,5 \%)$ (figure 3$)$.

Figure 3 : Nombre de spécimens positifs soumis aux laboratoires de Santé publique Ontario par type de virus et par année, de 2009 à 2014 (par 100000 habitants) $^{\mathrm{a}}$

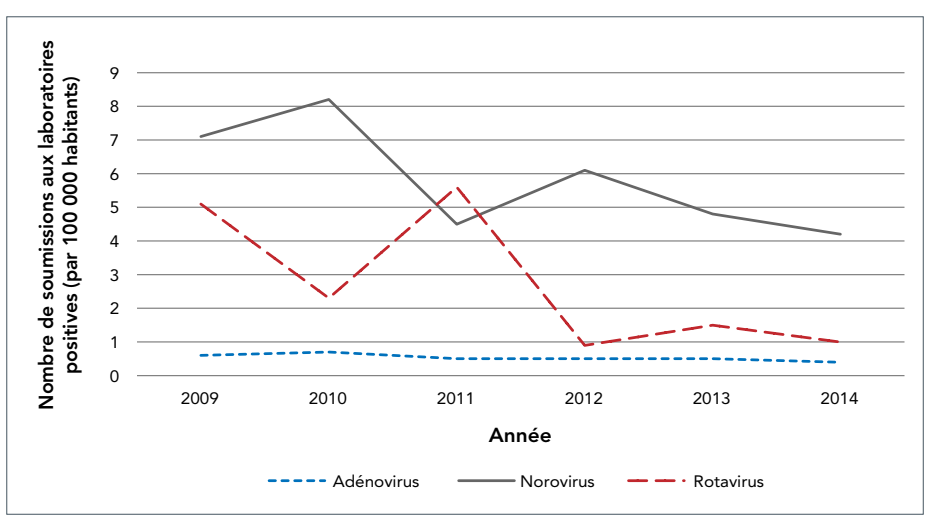

a Types de virus supplémentaires (astrovirus, sapovirus, autres Picornaviridae et autres Caliciviridae) retirés en raison de taux inférieurs à 0,10 spécimen positif pour 100000 habitants Remarque : Le vaccin antirotavirus (Rotarix ${ }^{\mathrm{MD}}$ ) a été ajouté au calendrier de vaccination de routine de l'Ontario en 2011

Les femmes $(40,0 \%)$ ont présenté plus de gastroentérite virale positive ( $n=7312$ ) que les hommes $(32,4 \%)$; toutefois, un grand pourcentage des échantillons présentaient des renseignements incomplets sur le sexe (27,6\%). Lorsqu'on a limité cette analyse aux échantillons positifs aux norovirus $(n=4633)$, on a répété cette tendance.

Le groupe d'âge des 65 ans et plus affichait le plus grand nombre de déclarations positives pour la gastroentérite virale, suivi du groupe d'âge des 0 à 4 ans; $93,5 \%$ et $15,3 \%$ de ces déclarations positives pour la gastroentérite virale étaient positives pour les norovirus, respectivement (figure 4). Parmi toutes les soumissions positives de gastroentérite virale, $19 \%$ ne contenaient pas de renseignements sur l'âge.

Au total, 62,3\% ( $n=4559 / 7$ 312) des échantillons positifs pour la gastroentérite virale ont été associés à des éclosions. Parmi les échantillons de l'éclosion, les foyers de soins de longue durée étaient le type d'emplacement le plus souvent touché, suivis des hôpitaux, des maisons de retraite, des garderies et des restaurants. La saisonnalité des soumissions aux laboratoire positives aux norovirus, ventilées par mois et par année, était étroitement associée à la saisonnalité des éclosions de norovirus (figure 2).

Appels de télésanté : Au total, 320834 appels de télésanté ont été enregistrés pour des maladies liées à la gastroentérite aiguë au cours de la période de 2009 à 2014. Parmi ces appels, $36,6 \%$ étaient dus à des vomissements comme principal motif 
Figure 4 : Activité de gastroentérite virale attribuée aux norovirus par groupe d'âge de 2019 à 2014a

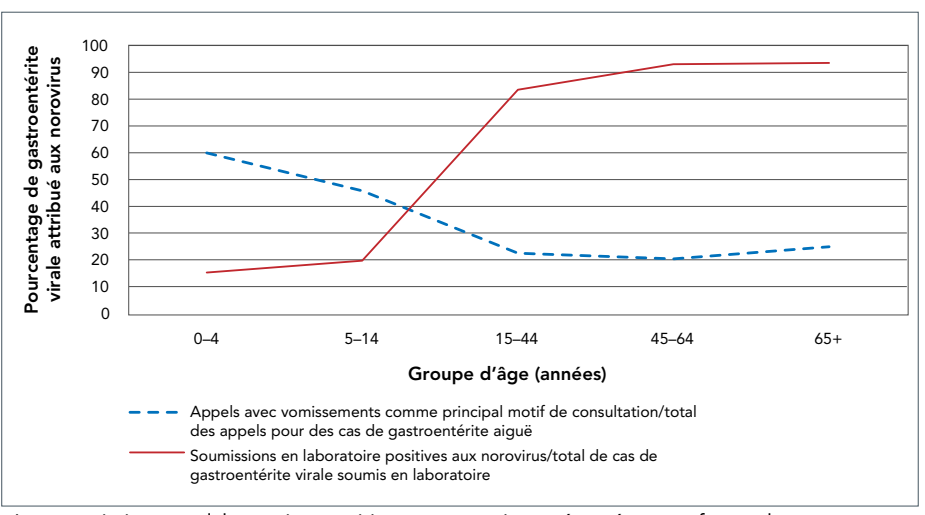

Les soumissions aux laboratoires positives aux norovirus présentées sous forme de pourcentage des spécimens positifs aux gastroentérites virales soumis à Santé publique Ontario; les motifs principaux de consultation concernant les vomissements (vomissements, diarrhée, vomissements et diarrhée) des appels à Télésanté Ontario en pourcentage de tous les appels liés aux gastroentérites aiguës

de consultation. Le pourcentage d'appels liés aux gastroentérites aiguës attribués aux vomissements comme principal motif de consultation a fluctué entre $31 \%$ et $41 \%$ entre 2009 et 2014 (figure 5).

Figure 5 : Pourcentage d'appels liés à la gastroentérite aiguë à Télésanté Ontario attribués aux vomissements comme principal motif de consultation ${ }^{a}$ par année, de 2009 à 2014

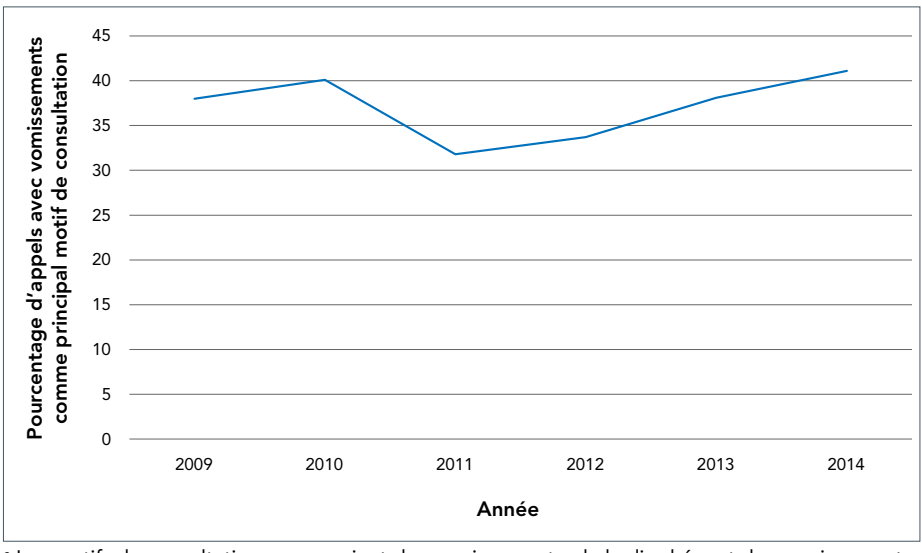

Les motifs de consultation concernaient des vomissements, de la diarrhée, et des vomissements et diarrhée

Les appels de télésanté étaient plus fréquents chez les femmes (62,6\% des appels pour les gastroentérites aiguës); les appels chez les hommes représentaient $35,9 \%$ et $1,5 \%$ chez les personnes de sexe inconnu ou non indiqué. Lors de l'analyse des appels avec vomissements comme principal motif de consultation, cette tendance s'est répétée.

Le groupe des 15 à 44 ans comptait le plus grand nombre d'appels de télésanté liés aux gastroentérites aiguës, soit 131271 (40,9\%) entre 2009 et 2014 . Toutefois, dans le cas des appels avec vomissements comme principal motif de consultation, les volumes d'appels les plus élevés
( $n=60058$ appels) ont été enregistrés pour le groupe des 0 à 4 ans. Le groupe des 65 ans et plus a toujours eu le plus faible nombre d'appels. Les groupes d'âge les plus jeunes (0 à ans, 5 à 14 ans) affichaient des pourcentages plus élevés d'appels liés aux gastroentérites aiguës avec vomissements comme principal motif de consultation comparativement aux groupes plus âgés (15 à 44 ans, 45 à 64 ans, 65 ans et plus) (figure 4). Moins de $1 \%$ des appels avec vomissements et gastroentérite aiguë comme principal motif de consultation avaient des renseignements manquants sur l'âge.

\section{Discussion}

Les norovirus ont été la cause la plus courante de cas et d'éclosions de gastroentérite virale en Ontario au cours des années 2009 à 2014. Ces travaux confirment des recherches antérieures qui ont permis de déterminer que les norovirus sont la cause la plus courante des infections à gastroentérite virale et intestinales dans la collectivité $(1,14,21,22)$. L'ensemble de données sur les éclosions a montré que les norovirus représentaient $41,6 \%$ de toutes les éclosions de gastroentérite virale pendant la période de l'étude, l'ensemble de données des laboratoires a montré que les norovirus représentaient $63,4 \%$ de toutes les soumissions laboratoires de gastroentérite virale, et l'ensemble de données de télésanté a montré que $36,6 \%$ de tous les appels concernant une gastroentérite aiguë avaient des vomissements comme principal motif de consultation.

Les données sur les éclosions de 2009 à 2014 ont démontré que les foyers de soins de longue durée étaient le type d'établissement le plus souvent impliqué dans les cas de gastroentérite virale et norovirus (figure 1). Cela n'était pas inattendu en raison de l'incidence plus élevée de la gastroentérite virale chez les adultes âgés; le virus cause de façon disproportionnée des maladies plus graves dans les populations vulnérables, comme les personnes âgées, les jeunes enfants et les personnes dont le système immunitaire est affaibli (1). Le nombre d'éclosions de gastroentérite virale par année dans les garderies a diminué après 2011 (figure 1). Ce résultat est probablement attribuable à l'introduction du vaccin Rotarix ${ }^{\mathrm{MD}}$ administré à l'âge de deux et quatre mois selon le calendrier de vaccination des enfants de l'Ontario en août 2011, ce qui a principalement eu une incidence sur les éclosions qui se sont produites dans les garderies (23). Le rotavirus est une maladie courante chez les enfants de moins de cinq ans, son principal symptôme étant la diarrhée. Par conséquent, la présence d'une infection par rotavirus dans ces données a probablement une incidence sur le nombre d'éclosions de gastroentérite virale chez les groupes de jeunes. D'autres études, tant en Ontario que dans des pays du monde entier, ont également signalé l'utilisation de la surveillance pour déterminer les diminutions de l'activité du rotavirus à la suite de l'introduction du vaccin (23-25). 
Les éclosions et les données de laboratoires ont montré une augmentation de l'activité des norovirus au-dessus de I'activité saisonnière normale pendant I'hiver 2009-2010 (figure 2). Une augmentation au-dessus des niveaux saisonniers normaux d'activité des norovirus se produit habituellement avec l'introduction d'une nouvelle souche, principalement en raison du taux de mutation rapide du virus et du manque d'immunité collective dans la population $(22,26)$. L'introduction de nouvelles souches peut entraîner des variations saisonnières et une augmentation du nombre d'éclosions (27). Ceci est probablement le résultat de l'émergence de deux nouvelles souches, la souche Gll.4 de la Nouvelle-Orléans, qui a touché des pays du monde entier, et une souche Gll.12 $(28,29)$. La souche Gll.4 de la Nouvelle-Orléans a causé de nombreuses éclosions et était si répandue qu'elle a été détectée en grand nombre jusqu'en 2013; cela pourrait également expliquer le pic plus élevé observé à la figure 2 pour la saison 2011-2012 (30).

Les deux pathogènes les plus fréquemment signalés étaient les norovirus et le rotavirus. Les norovirus ont tendance à toucher de façon disproportionnée les personnes très jeunes (de moins de cinq ans) et plus âgées (65 ans et plus) comparativement aux personnes d'âge moyen en bonne santé. Les éclosions sont très fréquentes dans les régions à forte densité, comme les garderies, les maisons de retraite et les foyers de soins de longue durée $(4,31)$. Le rotavirus a un résultat semblable en ce sens qu'il touche de façon disproportionnée les jeunes enfants (de moins de cinq ans), ce qui entraîne également des éclosions dans les garderies, les écoles maternelles, etc. $(1,23)$. Les personnes plus âgées ( 65 ans et plus) et celles qui vivent dans des foyers de soins de longue durée peuvent avoir un plus grand besoin de soins médicaux aigus et être plus susceptibles de faire l'objet de prélèvements d'échantillons qui sont soumis aux autorités de la santé publique, ce qui pourrait expliquer ces constatations. Il convient de noter que la différence entre les échantillons de norovirus et les échantillons positifs au rotavirus existait même avant l'introduction du vaccin Rotarix dans le calendrier de vaccination des enfants de l'Ontario. De plus, nos analyses ont clairement démontré la diminution aiguë des cas de rotavirus à la suite de l'introduction du vaccin Rotarix en 2011. À partir de 2012, les spécimens positifs en laboratoire étaient presque entièrement des norovirus.

La différence dans les volumes d'appels de télésanté pour la gastroentérite aiguë et les vomissements comme principal motif de consultation pour les groupes d'âge de 15 à 44 ans et de 0 à 4 ans, respectivement, est probablement influencée par le rotavirus. Étant donné que la maladie touche de façon disproportionnée les jeunes, elle entraînerait un volume d'appels plus élevé pour le groupe des 0 à 4 ans. Il est probable que de nombreux parents qui téléphonent au service de télésanté s'inquiètent pour leurs enfants. Le groupe des 15 à 44 ans avait probablement le plus grand nombre d'appelants pour la gastroentérite aiguë en raison des diverses maladies qui touchent cette population et de leur préférence pour les soins virtuels.
Bien que les ensembles de données aient donné un aperçu de l'activité des cas de gastroentérite virale et de norovirus en Ontario, il y avait un désavantage évident, soit le manque de données communautaires (i.e. les données sur les personnes qui souffrent d'une maladie et qui restent dans leur domicile). Les ensembles de données de laboratoires et sur les éclosions sont biaisés vers les milieux institutionnels principalement parce que, à l'extérieur des milieux institutionnels, il n'est pas obligatoire de déclarer les norovirus et les autres gastroentérites virales aux autorités de la santé publique de l'Ontario. De plus, de nombreux cas de gastroentérite virale (les norovirus en particulier) sont sous-déclarés (32). L'inclusion de données de télésanté dans cette étude aide à combler cette lacune en ce sens qu'elle recueille principalement des données communautaires qui ne sont pas bien représentées par les éclosions ou les données de laboratoires. Les données syndromiques sont connues pour leur capacité à réduire la sous-déclaration et représentent un pourcentage plus élevé de la population (33). Par conséquent, l'inclusion des données de télésanté permet de mieux comprendre l'activité des cas de gastroentérite virale en Ontario et de réduire le biais.

Les données syndromiques sont de plus en plus courantes et fréquemment utilisées dans les pratiques de santé publique en raison de leur éventail d'avantages. Elles sont disponibles en temps opportuns, peuvent détecter des menaces nouvelles ou émergentes, complètent les données des systèmes de surveillance traditionnels et ne sont pas spécifiques (34). Les données de télésanté sont particulièrement bénéfiques, car elles représentent l'une des options de données syndromiques les plus opportunes disponibles; les lignes d'assistance de télémédecine sont l'un des premiers points de soins médicaux pour les patients symptomatiques (34). Ces données sont également connues pour leur disponibilité en temps réel et leur facilité d'accès. Toutefois, les données de télésanté ne sont pas aussi précises que d'autres sources et ne peuvent pas nécessairement être utilisées pour détecter des éclosions précises ou graves (34). Dans cette étude, les données de télésanté n'ont pas besoin d'être précises parce que les appels concernant des symptômes gastro-intestinaux non spécifiques fournissent l'alerte précoce de la maladie requise pour le système conçu, et sera le moyen le plus efficace d'observer les norovirus et les maladies gastro-intestinales en Ontario lorsqu'ils seront combinés aux données des laboratoires et des éclosions.

\section{Limites}

Cette étude comporte quelques limites notables. Dans l'ensemble de données sur les éclosions, il n'y avait pas de données liées à l'âge. Le type d'établissement a plutôt été utilisé comme indicateur d'âge. Par ailleurs, il y avait de nombreuses entrées " gastroentérite non spécifiée » et " gastroentérite autre » dans l'ensemble de données, qui contenaient probablement d'autres cas de norovirus, mais qui étaient inutilisables. De plus, il y avait un manque de déclaration normalisée des norovirus et des gastroentérites virales en Ontario. Seuls les cas institutionnels de norovirus doivent être 
signalés aux autorités de santé publique de l'Ontario (qui souffrent également d'une sous-déclaration et de retards). Par conséquent, les analyses des norovirus et de l'activité des gastroentérites virales sont difficiles en raison des lacunes dans les données, ainsi que des biais dans la déclaration des groupes d'âge à l'échelle de la province. Il est également important de noter qu'il y a probablement une surreprésentation dans l'ensemble de données de laboratoire. Cet ensemble de données comprend à la fois des échantillons d'éclosion et des échantillons sporadiques; en cas d'éclosion, un ou plusieurs échantillons peuvent être soumis. Par conséquent, cette étude n'est pas en mesure de décrire précisément le fardeau lié aux norovirus sporadiques en Ontario, et on met probablement davantage l'accent sur les données liées aux éclosions. Enfin, les appels de télésanté peuvent inclure des causes non virales et contenir des appels en double; toutefois, il n'a pas été possible de les stratifier dans l'ensemble de données. De plus, on a supposé que les vomissements étaient le principal symptôme des norovirus pour les analyses de télésanté, ce qui a probablement exclu de nos résultats d'autres appels liés aux norovirus. Chaque ensemble de données présentait des limites sur le plan de la population représentative; toutefois, lorsqu'on l'a combiné, on a produit un résumé global de l'épidémiologie des norovirus en Ontario pendant la période de 2009 à 2014.

\section{Conclusion}

Cette étude décrit l'épidémiologie de la gastroentérite virale et plus précisément, des norovirus dans la province de l'Ontario, au Canada, entre 2009 et 2014. Notre étude démontre que les norovirus sont très répandus et qu'ils sont la cause la plus dominante de gastroentérite virale dans la province. Nos résultats sont conformes à ceux d'études internationales semblables, qui démontrent que les norovirus sont la principale cause de gastroentérite virale (1-3). Bien qu'un vaccin ait été introduit en Ontario et dans des pays du monde entier pour atténuer l'infection par le rotavirus, il n'existe pas de vaccin contre les norovirus. L'introduction d'interventions préventives, comme un vaccin, est idéale; cependant, d'autres mesures de santé publique, comme de nouvelles techniques de surveillance, sont également nécessaires pour éclairer les interventions en santé publique. Une combinaison de techniques de surveillance traditionnelles et nouvelles permettra de mieux saisir les données représentatives de la population ontarienne et de réduire les biais en matière de surveillance. II faudrait envisager d'autres techniques pour aider à estimer les lacunes dans les connaissances sur le fardeau des norovirus, comme les norovirus sporadiques (35).

\section{Déclaration des auteurs}

S. L. H. - Conceptualisation, obtention d'ensembles de données, méthodologie, analyse et interprétation des données, rédaction et révision du manuscrit

A. P. - Participation à l'étude conçue, à la méthodologie, à la révision
Tous les auteurs ont contribué aux révisions de la méthodologie et de l'article et ont lu et approuvé la version finale de l'article.

Le contenu de l'article et les points de vue qui y sont exprimés n'engagent que les auteurs et ne correspondent pas nécessairement à ceux du gouvernement du Canada.

\section{Intérêts concurrents}

Aucun.

\section{Remerciements}

Nous sommes reconnaissants de l'aide fournie par Public Health England tout au long de cette étude. De plus, il faut remercier W. Sears, de I'Université de Guelph, pour son aide dans le cadre des analyses de cette étude.

\section{Financement}

Ce travail a été appuyé par l'Université de Guelph.

\section{Références}

1. Patel MM, Hall AJ, Vinjé J, Parashar UD. Noroviruses: a comprehensive review. J Clin Virol 2009;44(1):1-8. DOI PubMed

2. Morton VK, Thomas MK, McEwen SA. Estimated hospitalizations attributed to norovirus and rotavirus infection in Canada, 20062010. Epidemiol Infect 2015;143(16):3528-37. DOI PubMed

3. Yen $\mathrm{C}, \mathrm{Hall}$ AJ. Challenges to estimating norovirus disease burden. J Pediatric Infect Dis Soc 2013;2(1):61-2. DOI PubMed

4. Karst SM. Pathogenesis of noroviruses, emerging RNA viruses. Viruses 2010;2(3):748-81. DOI PubMed

5. Glass RI, Parashar UD, Estes MK. Norovirus gastroenteritis. N Engl J Med 2009;361(18):1776-85. DOI PubMed

6. Teunis PF, Moe CL, Liu P, Miller SE, Lindesmith L, Baric RS, Le Pendu J, Calderon RL. Norwalk virus: how infectious is it? J Med Virol 2008;80(8):1468-76. DOI PubMed

7. Kaplan JE, Gary GW, Baron RC, Singh N, Schonberger LB, Feldman R, Greenberg HB. Epidemiology of Norwalk gastroenteritis and the role of Norwalk virus in outbreaks of acute nonbacterial gastroenteritis. Ann Intern Med 1982;96(6 Pt 1):756-61. DOI PubMed

8. Thomas MK, Murray R, Flockhart L, Pintar K, Pollari F, Fazil A Nesbitt A, Marshall B. Estimates of the burden of foodborne illness in Canada for 30 specified pathogens and unspecified agents, circa 2006. Foodborne Pathog Dis 2013;10(7):639-48. DOI PubMed

9. Hall AJ, Lopman BA, Payne DC, Patel MM, Gastañaduy PA, Vinjé J, Parashar UD. Norovirus disease in the United States. Emerg Infect Dis 2013;19(8):1198-205. DOI PubMed 
10. Burke RM, Mattison C, Marsh Z, Shioda K, Donald J, Salas SB, Naleway AL, Biggs C, Schmidt MA, Hall AJ. Norovirus and other viral causes of medically attended acute gastroenteritis across the age spectrum: Results from the Medically Attended Acute Gastroenteritis Study in the United States. Clin Infect Dis. 2021;73(6):e913-20. DOI

11. Cremon C, De Giorgio R, Giovanni B. Norovirus gastroenteritis. N Engl J Med 20101362(6):577-8. DOI PubMed

12. Tarr GA, Pang $X L$, Zhuo $R$, Lee BE, Chui L, Ali S, Vanderkooi OG, Michaels-lgbokwe C, Tarr PI, MacDonald SE, Currie G, MacDonald J, Kim K, Freedman SB. Attribution of pediatric acute gastroenteritis episodes and emergency department visits to norovirus genogroups I and II. J Infect Dis 2021;223(3):452-61. DOI PubMed

13. Esposito S, Principi N. Norovirus vaccine: priorities for future research and development. Front Immunol 2020;11:1383. DOI PubMed

14. Hall AJ, Rosenthal M, Gregoricus N, Greene SA, Ferguson J, Henao OL, Vinjé J, Lopman BA, Parashar UD, Widdowson MA. Incidence of acute gastroenteritis and role of norovirus, Georgia, USA, 2004-2005. Emerg Infect Dis 2011;17(8):1381-8. DOI PubMed

15. Statistique Canada. Estimations de la population au 1 er juillet, par âge et sexe. Ottawa (ON) : Gouvernement du Canada; 2021 (accédé 2021-04-10). https://www150.statcan.gc.ca/t1/tbl1/fr/tv.action?pid= 1710000501\&pickMembers\%5B0\%5D=1.7\&pickMembers\%5B1\%5D $=2.1$ \&request_locale $=\mathrm{fr}$

16. Freedman SB, Xie J, Lee BE, Ali S, Pang X-L, Chui L, Zhuo R, Vankerkooi OG, Tellier R, Funk AL, Tarr Pl, Alberta Provincial Pediatric EnTeric Infection Team (APPETITE). Microbial etiologies and clinical characteristics of children seeking emergency department care due to vomiting in the absence of diarrhea. Clin Infect Dis 2021:ciab451. (Publié en ligne avant impression). DOI

17. Ministère de l'Éducation. Trouver des services de garde d'enfants agréés. Toronto (ON) : Gouvernement de l'Ontario; 2019 (accédé 2021-04-10). https://www.iaccess.gov.on.ca/LCCWWeb/childcare/ search. $x$ html?commonTask $=Y$

18. Ministère du Solliciteur général. Services correctionnels : Établissements - emplacements et heures de visite. Toronto (ON) : Gouvernement de l'Ontario; 2020 (accédé 2021-04-10). https://www.mcscs.jus.gov.on.ca/french/corr_serv/facilitieslocations andvisitinghours/facilities_fr.html

19. Ontario Long Term Care Association. About long-term care in Ontario: Facts and figures. Toronto (ON): OLTCA; 2019 (accédé 2021-04-10). http://www.oltca.com/oltca/OLTCA/LongTermCare/ OLTCA/Public/LongTermCare/FactsFigures.aspx

20. Statistique Canada. Estimations démographiques annuelles par région économique, âge et sexe, basées sur la Classification géographique type (CGT) 2011, inactif. Ottawa (ON) : Gouvernement du Canada; 2021 (accédé 2021-04-10). https://www150.statcan.gc.ca/t1/tbl1/fr/cv.action?pid=1710008101 \&request_locale $=\mathrm{fr}$

21. Loveridge P, Cooper D, Elliot AJ, Harris J, Gray J, Large S, Regan M, Smith GE, Lopman B. Vomiting calls to NHS Direct provide an early warning of norovirus outbreaks in hospitals. J Hosp Infect 2010;74(4):385-93. DOI PubMed

22. Gastañaduy PA, Hall AJ, Curns AT, Parashar UD, Lopman BA. Burden of norovirus gastroenteritis in the ambulatory setting-United States, 2001-2009. J Infect Dis 2013;207(7):1058-65. DOI PubMed
23. Wilson SE, Rosella LC, Wang J, Le Saux N, Crowcroft NS, Harris T, Bolotin S, Deeks SL. Population-level impact of Ontario's infant rotavirus immunization program: evidence of direct and indirect effects. PLoS One 2016;11(5):e0154340. DOI PubMed

24. Thomas SL, Walker JL, Fenty J, Atkins KE, Elliot AJ, Hughes HE, Stowe J, Ladhani S, Andrews NJ. Impact of the national rotavirus vaccination programme on acute gastroenteritis in England and associated costs averted. Vaccine 2017;35(4):680-6. DOI PubMed

25. Bawa Z, Elliot AJ, Morbey RA, Ladhani S, Cunliffe NA, O'Brien SJ, Regan M, Smith GE. Assessing the likely impact of a rotavirus vaccination program in England: the contribution of syndromic surveillance. Clin Infect Dis 2015;61(1):77-85. DOI PubMed

26. Karst SM, Baric RS. What is the reservoir of emergent human norovirus strains? J Virol 2015;89(11):5756-9. DOI PubMed

27. Allen DJ, Adams NL, Aladin F, Harris JP, Brown DW. Emergence of the GIl-4 Norovirus Sydney2012 strain in England, winter 2012-2013. PLoS One 2014;9(2):e88978. DOI PubMed

28. Vega E, Vinjé J. Novel GII.12 norovirus strain, United States, 2009-2010. Emerg Infect Dis 2011;17(8):1516-8. DOI PubMed

29. Yen C, Wikswo ME, Lopman BA, Vinjé J, Parashar UD, Hall AJ. Impact of an emergent norovirus variant in 2009 on norovirus outbreak activity in the United States. Clin Infect Dis 2011;53(6):568-71. DOI PubMed

30. Medici MC, Tummolo F, Grazia S, Calderaro A, Conto F, Terio V, Chironna M, Bonura F, Pucci M, Bányai K, Martella V, Giammanco GM. Epidemiological dynamics of norovirus Gll.4 variant New Orleans 2009. J Gen Virol 2015;96(9):2919-27. DOI PubMed

31. Said MA, Perl TM, Sears CL. Healthcare epidemiology: gastrointestinal flu: norovirus in health care and long-term care facilities. Clin Infect Dis 2008;47(9):1202-8. DOI PubMed

32. Tam CC, Rodrigues LC, Viviani L, Dodds JP, Evans MR, Hunter PR, Gray JJ, Letley LH, Rait G, Tompkins DS, O'Brien SJ; IID2 Study Executive Committee. Longitudinal study of infectious intestinal disease in the UK (IID2 study): incidence in the community and presenting to general practice. Gut 2012;61(1):69-77. DOl PubMed

33. Silva AD, Evangelista MS. Syndromic surveillance: etiologic study of acute febrile illness in dengue suspicious cases with negative serology. Brazil, Federal District, 2008. Rev Inst Med Trop São Paulo 2010;52(5):237-42. DOI

34. French Institute for Public Health Surveillance. Triple-S Project (Syndromic Surveillance System in Europe). Guidelines for designing and implementing a syndromic surveillance system. InVS; 2013. https://webgate.ec.europa.eu/chafea_pdb/assets/files/ pdb/20091112/20091112_d08_giss_en_ps.pdf

35. Vanderkooi OG, Xie J, Lee BE, Pang XL, Chui L, Payne DC, MacDonald J, Ali S, MacDonald S, Drews S, Osterreicher L, Kim K, Freedman SB; Alberta Provincial Pediatric EnTeric Infection TEam (APPETITE) and Pediatric Emergency Research Canada (PERC). A prospective comparative study of children with gastroenteritis: emergency department compared with symptomatic care at home. Eur J Clin Microbiol Infect Dis 2019;38(12):2371-9. DOI 


\section{Appendice}

\section{Système intégré d'information sur la santé publique}

L'ensemble de données du Système intégré d'information sur la santé publique (SIISP) contient de l'information sur les éclosions confirmées de gastroentérite virale en Ontario signalées à la santé publique entre le 1er janvier 2009 et le 13 décembre 2014. En Ontario, il est obligatoire que toutes les maladies à déclaration obligatoire (depuis 2018, ces maladies sont maintenant appelées " maladies d'intérêt en santé publique ») soient soumises à la base de données $(1,2)$. Les bureaux de santé publique locaux sont responsables de recueillir des renseignements sur toutes les maladies à déclaration obligatoire et de les entrer dans le SIISP, dans le cadre de la surveillance provinciale et fédérale.

Toutes les éclosions présentées dans l'ensemble de données sont institutionnelles (i.e. qu'elles sont survenues dans des maisons de soins de longue durée, des maisons de retraite, des établissements de soins pour enfants, des établissements correctionnels, etc.). Les renseignements supplémentaires trouvés dans le SIISP comprennent la méthode d'exposition (si elle est déterminée), l'agent étiologique (s'il est déterminé), le bureau de santé publique, l'état confirmé de la maladie, la date à laquelle l'éclosion a été déclarée par le médecin hygiéniste, la date à laquelle l'éclosion a été déclarée terminée par le médecin-hygiéniste, la date du début initial (heure du cas de référence) et la date du début final (heure du dernier cas). Les dates de début (heure du cas de référence) ont été utilisées pour les analyses de cette étude, et la plage entre la date de début (heure du cas de référence) et la date de début (heure du dernier cas) a été utilisée pour calculer la durée moyenne de l'éclosion de gastroentérite virale et de norovirus.

Bien que les données du SIISP soient continuellement mises à jour, les autorités de la santé publique sont tenues d'entrer toutes les éclosions de l'année écoulée au plus tard en août de chaque année, ce qui entraîne des retards. II faut noter qu'il n'est pas nécessaire d'obtenir la confirmation du laboratoire pour qu'une éclosion soit entrée dans l'ensemble de données, et lorsque la confirmation du laboratoire est présente, tous les cas associés à une éclosion ne sont pas testés en laboratoire.

\section{Laboratoires de Santé publique Ontario}

L'ensemble de données des laboratoires de Santé publique Ontario (LSPO) représente tous les échantillons soumis à Santé publique Ontario (SPO) entre le $1^{\mathrm{er}}$ janvier 2009 et le 31 décembre 2014 aux fins d'analyse en Ontario. Les données représentent des échantillons qui ont été soumis à SPO de patients atteints de norovirus ou de rotavirus présumés (i.e. symptômes de vomissements et/ou de diarrhée). Les échantillons sont soumis par des professionnels de la santé sous forme d'échantillons de selles et sont testés par réaction en chaîne de la polymérase, microscopie électronique ou test immunochromatographique. L'ensemble de données fournit des renseignements sur l'âge, le sexe et le bureau de santé publique, ainsi que les dates auxquelles les échantillons ont été prélevés et entrés dans l'ensemble de données par la suite. Pour les analyses, les dates de prélèvement des échantillons ont été utilisées (i.e. que les résultats représentent la date à laquelle les échantillons ont été prélevés sur la personne malade par le professionnel de la santé à des fins d'analyse). II est à noter que ce ne sont pas toutes les demandes d'analyse de gastroentérite virale des établissements et des médecins qui sont testées par SPO en Ontario; les échantillons peuvent aussi être envoyés à d'autres laboratoires privés en Ontario ou en double à plusieurs laboratoires. Par conséquent, les échantillons de gastroentérite virale confirmés en laboratoire de l'Ontario ne sont pas tous saisis dans cet ensemble de données.

\section{Télésanté Ontario}

L'ensemble de données de Télésanté Ontario représente tous les appels téléphoniques effectués par des résidents de l'Ontario entre le $1^{\text {er }}$ janvier 2009 et le 31 décembre 2014 ayant comme principal motif de consultation les symptômes gastro-intestinaux. Télésanté Ontario est un service téléphonique confidentiel offert en tout temps qui est en service depuis 2001 et qui est accessible à tous (il n'est pas nécessaire d'avoir un numéro de carte d'assurance-maladie). Le service est exploité par la Sykes Assistance Services Corporation, qui a un contrat avec le ministère de la Santé et des Soins de longue durée de l'Ontario. Une personne peut appeler la ligne d'assistance pour n'importe quelle raison, et un infirmier répond et fournit des conseils médicaux de base et oriente l'appelant vers un plan d'action approprié (i.e. se rendre immédiatement à la salle des urgences, consulter un médecin de famille très rapidement ou dans le jour suivant, ou conseiller des soins personnels). La ligne d'assistance aide à alléger la pression sur les services d'urgence et les bureaux de médecin, tout en fournissant gratuitement des conseils médicaux à des millions d'Ontariennes et d'Ontariens. L'ensemble de données de Télésanté Ontario fournit des renseignements sur la date et l'heure de l'appel, le motif de consultation principal de l'appelant (raison principale de l'appel), les prochaines étapes suggérées par l'infirmier, l'âge et le sexe de l'appelant et l'emplacement (ville) d'où l'appel a été émis.

Dans le cadre de cette étude, les appels faits à Télésanté Ontario sont appelés " gastroentérite aiguë " plutôt que " gastroentérite virale ", comme c'est le cas dans les ensembles de données du SIISP et de SPO, en raison du fait qu'ils sont moins précis et qu'ils englobent toutes les causes de gastroentérite aiguë, et non pas uniquement les étiologies virales. Par ailleurs, les appels à Télésanté Ontario pour des maladies qui semblent provenir des norovirus n'ont pas été confirmés comme étant des norovirus. Par conséquent, les « vomissements comme motif principal de consultation » sont utilisés comme substitut des norovirus et des maladies qui semblent provenir des norovirus, et le fardeau des appels liés aux gastroentérites aiguës ne peut être attribué strictement aux norovirus, mais plutôt aux norovirus et aux maladies qui semblent provenir des norovirus. 